\title{
Purification of Tropomyosin BR-3 and 5NM1 and Characterization of Their Interactions with Actin
}

\author{
Nikolett Kis-Bicskei, ${ }^{1}$ Andrea Vig,, Miklós Nyitrai, ${ }^{1,2,3}$ Beáta Bugyi, ${ }^{1 *}$ and Gábor C. Talián ${ }^{1 *}$ \\ ${ }^{1}$ Department of Biophysics, University of Pécs, Medical School, Szigeti u. 12, Pécs, Hungary \\ ${ }^{2}$ Szentágothai Research Center, Ifjúság u. 34, Pécs, Hungary \\ ${ }^{3}$ Hungarian Academy of Sciences, Office for Subsidized Research Units, Budapest, Nádor u. 7, Hungary
}

Received 3 May 2013; Revised 17 August 2013; Accepted 4 September 2013

Monitoring Editor: Christophe Ampe

Tropomyosins were first identified in neuronal systems in 1973. Although numerous isoforms were found and described since then, many aspects of their function and interactions remained unknown. Tropomyosin isoforms show different sorting pattern in neurogenesis. As one example, TM5NM1/2 is present in developing axons, but it is replaced by $\mathrm{TMBr}-3$ in mature neurons, suggesting that these tropomyosin isoforms contribute differently to the establishment of the functional features of the neuronal actin networks. We developed a method for the efficient purification of $\mathrm{TMBr}-3$ and TM5NM1 as recombinant proteins using bacterial expression system and investigated their interactions with actin. We found that both isoforms bind actin filaments, however, the binding of TM5NM1 was much stronger than that of TMBr-3. TMBr-3 and TM5NM1 modestly affected actin assembly kinetics, in an opposite manner. Consistently with the higher affinity of TM5NM1 it inhibited actin filament disassembly more efficiently than $\mathrm{TMBr}-3$. Similarly to other previously studied tropomyosins TM5NM1 inhibited the Arp2/3 complex-mediated actin assembly. Notably, TMBr-3 did not influence the Arp2/3 complex-mediated polymerization. This is a unique feature of $\mathrm{TMBr}-3$, since so far it is the only known tropomyosin supporting the activity of the Arp2/3 complex, indicating that $\mathrm{TMBr}-3$ may colocalize and work simultaneously with Arp2/3 complex in neuronal cells. $\odot 2013$ Wiley Periodicals, Inc.

Key Words: actin; tropomyosin; actin nucleation factors; purification; fluorescence spectroscopy

\footnotetext{
*Address correspondence to: Gábor C. Talián; Department of Biophysics, University of Pécs, Medical School, Szigeti str. 12, Pécs H-7624, Hungary. E-mail: gabor.c.talian@aok.pte.hu or Beáta Bugyi; Department of Biophysics, University of Pécs, Medical School, Szigeti str. 12, Pécs H-7624, Hungary. E-mail: beata. bugyi@aok.pte.hu

Published online 00 Month 2013 in Wiley Online Library (wileyonlinelibrary.com).
}

\section{Introduction}

$T$ The microfilament system orchestrates a great number of diverse cellular processes, including the establishment cell geometry, cell motility, intracellular transport, contractility, cytokinesis and transformation. Structurally, the basic building blocks of microfilaments are actin and tropomyo$\sin$ (TM) [Vindin and Gunning, in press]. Both actin and tropomyosin exist in several isoforms, which assemble into distinct compartments with different molecular composition, morphology, and function [Gunning et al., 1998; Perrin and Ervasti, 2010].

Tropomyosins are elongated, rod-shaped molecules with extensive $\alpha$-helical conformation, forming parallel-chained coiled-coil dimers. The individual dimers assemble into helical polymers in a head-to-tail manner, cooperatively associating along the longitudinal axis of the actin filament on both sides [Flicker et al., 1982; Coulton et al., 2008]. Tropomyosins are present in all animal and fungal cells. In mammals four tropomyosin genes are known that produce more than 40 different mRNAs and about 25 protein isoforms [Gunning et al., 2005, 2008]. Traditionally, tropomyosin isoforms involved in the contractile apparatus of muscle cells are referred to as muscle isoforms, while isoforms associated to the cell cytoskeleton are referred to as nonmuscle or cytoskeletal tropomyosins. The expression and localization of tropomyosin isoforms are strictly regulated at both tissue and subcellular level, and often depends on the developmental state as well [Gunning et al., 2005, 2008]. Since the first discovery of TMs in the nervous system several neuronal isoforms have been described [Fine et al., 1973]. The TMBr-1, 2, and 3 are encoded by the $\alpha$-TM gene [Lees-Miller et al., 1990]. TMBr-2 and 3 are short, while $\mathrm{TMBr}-1$ is a long isoform, as characterized by the use of exon $1 \mathrm{a}$ and $2 \mathrm{~b}$ or exon $1 \mathrm{~b}$ at the $\mathrm{N}$-terminus, respectively (Fig. 1A). The $\gamma$-Tm gene also encodes tropomyosin isoforms present in neurons, like TM5NM1 and TM5NM2, both of them are short isoforms, carrying exon 1b (Fig. 1A). These isoforms exhibit different subcellular 
A

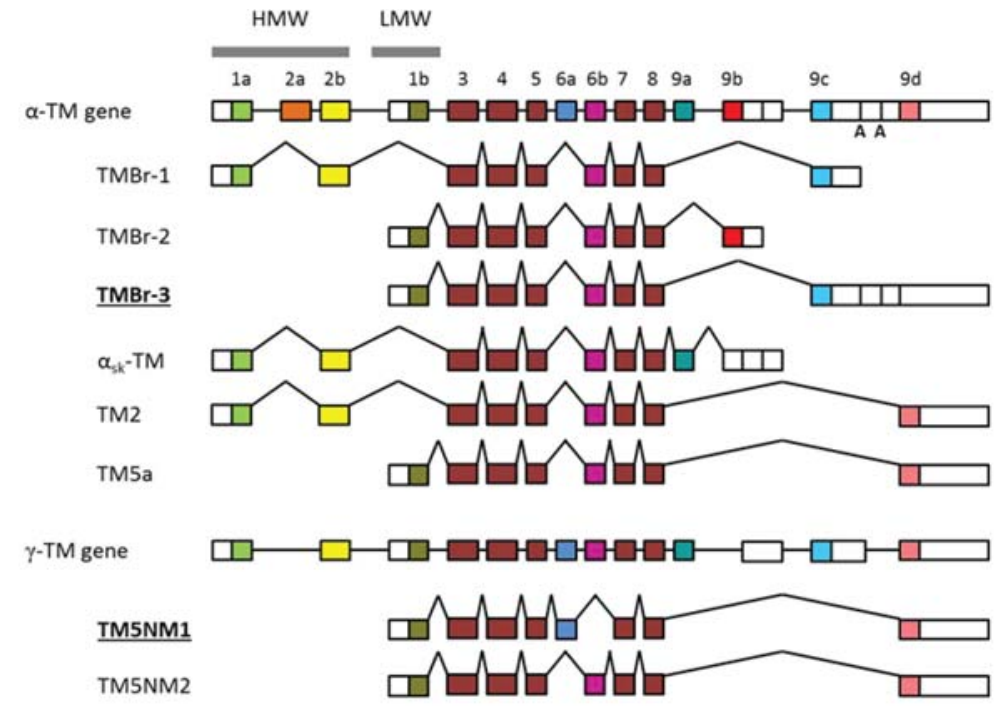

B

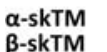

TM5NM1 - DQNLKCLSAABEKYSQKKBDKYBBEIK ILTDKLKBABTRABFABRSVAKLBK TIDDLBDKLKCTKBGHLCTQRMLDQTLLDLNBM

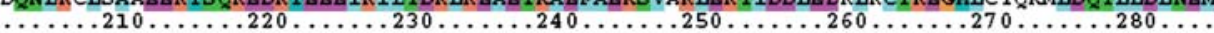

C

$\mathrm{MW}, \mathrm{kDa}$

66.2

45

35

25

L

$\mathrm{O}$

$\mathrm{R}$
123

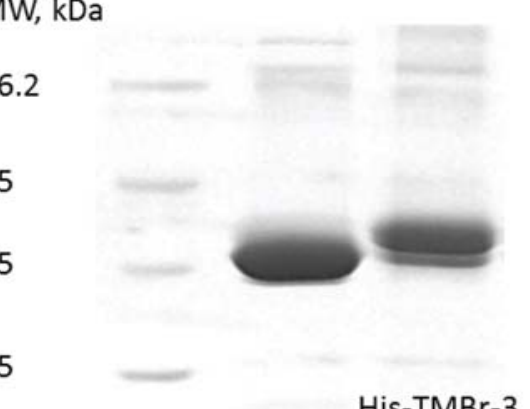

123

$\mathrm{MW}, \mathrm{kDa}$

66.2

45

35

25

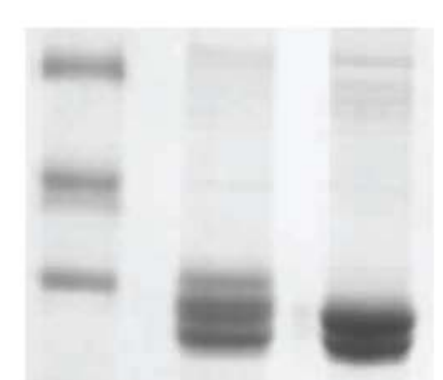

His-TM5NM1

TM5NM1

Fig. 1. (A) Exon organization of different tropomyosin isoforms. HMW: high molecular weight tropomyosins, containing exon 1a and 2; LMW: low molecular weight tropomyosins, containing exon 1b. (B) Sequence alignment of different tropomyosin isoforms; from top to bottom: alpha skeletal TM (Oryctolagus cuniculus), beta skeletal TM (Oryctolagus cuniculus), TMBr-3 (Mus Musculus), TM5NM1 (Mus musculus). The sequences were aligned using ClustalX. (C) SDS-PAGE of the purified TMBr-3 and TM5NM1 proteins before and after TEV protease digestion. Lane 1: MW: molecular weight marker (Fermentas).

distributions and developmental profiles, as well, suggesting their functional specialization for different neuronal processes.

TM5NM1/2 is abundantly present in early rodent embryos at both mRNA and protein level at the site of the axon pole in the differentiating neurons, similarly to the 7 day old cultured primer cortical neurons [Hannan et al., 1995]. Some days later the TM5NM1/2 proteins exhibit a predominantly axonal localization. $\mathrm{TMBr}-3$ appears only in 
the 16-day rat embryo, its amount is very low at birth in the rat cerebellum, then continuously increases until the third postnatal week. In PC12 cells cultured on substratum the mRNA expression of $\mathrm{TMBr}-3$ highly correlated with the induction of the neuronal morphological differentiation [Weinberger et al., 1993]. Around the 15-17 prenatal days the TM5NM1/2 mRNA disappears from the axon, which coincides with the appearance of $\mathrm{TMBr}-3$. The isoform change between the TM5NM1/2 and TMBr-3 also happens at the protein level around the 17 embryonic day, resulting in the repositioning of TM5NM1/2 into the somatodendritic compartment of the mature neurons. Similar results were obtained in chicken, as well, indicating that such a switch in the isoform composition is conserved through the evolution of the higher vertebrates [Weinberger et al., 1996]. Further studies with more specialized antibodies have demonstrated that TM5NM2 localized to the axon shaft [Schevzov et al., 1997], while TM5NM1 to the outer region of the growth cone in the early embryos [Schevzov et al., 2005]. TMBr-3 was absent from the growth cones [Schevzov et al., 1997; Had et al., 1994] and its distribution in cultured embryonic neurons was diffuse, showing no association with specific microfilament structures [Stamm et al., 1993]. The results also suggested that the function of TM5NM1 and TMBr-3 was associated with the differentiation, growth, and maintenance of the specialized neuronal processes. In stably transfected B35 neuroepithelial cell clones overexpressing $\mathrm{TMBr}-3$ very few stress fibers, decreased cell surface and increased cell motility with lamellipodium formation was observed. In contrast, TM5NM1 had opposite effects on most of these processes which was dominantly counteracted by $\mathrm{TMBr}-3$ when cotransfected. Also, TMBr-3 and TM5NM1 differently regulated the access of actin to its binding partners, such as myosin and actin depolymerizing factor (ADF)/cofilin [Bryce et al., 2003]. Altogether, the above results indicate that these tropomyosin isoforms can define functionally distinct microfilament compartments.

Despite the extensive studies on tropomyosins, the physicochemical properties and the molecular bases of the isoform-specific functional differences are not well understood. To better understand the cellular functioning of TMBr-3 and TM5NM1 isoforms we aimed to characterize their interactions with actin using biophysical and biochemical approaches. Here we report the purification of the recombinant $\mathrm{TMBr}-3$ and TM5NM1 and the in vitro characterization of their interactions with actin. We showed that both isoforms bind actin filaments, however, the affinity of TM5NM1 was much higher than that of TMBr-3. Both tropomyosins have minor effect on actin polymerization; TMBr-3 slightly decreased the rate of actin assembly, while TM5NM1 slightly increased it. Consistently with the higher affinity of TM5NM1 it inhibited actin filament disassembly more effectively than TMBr-3. TM5NM1 inhibited the Actin-related protein (Arp) 2/3 complex-mediated actin assembly as detected for other previously studied tropomyosins. Interestingly, $\mathrm{TMBr}-3$ by not influencing the Arp2/3 complex-mediated polymerization seems to be a unique tropomyosin isoform, since so far it is the only known tropomyosin supporting the activity of the Arp2/3 complex.

\section{Results}

\section{Expression and Purification of $\mathrm{TMBr}-3$ and TM5NM1}

We produced the TMBr-3 and TM5NM1 tropomyosin isoforms as recombinant proteins in sufficient amount for in vitro characterization of their interactions with actin. Binding of tropomyosin isoforms to actin filaments can exhibit a high degree of cooperativity, in which the formation of so called head-to-tail interactions between the $\mathrm{N}$-, and C-termini of neighboring tropomyosin molecules are crucial. Minor modifications in the amino acid sequence can affect actin binding and consequently the function of tropomyosins. Therefore, we aimed to purify $\mathrm{TMBr}-3$ and TM5NM1 isoforms as recombinant proteins without any excess amino acids of the original sequences. The full length coding sequences of the corresponding mouse tropomyosin isoforms were used for protein production (Fig. 1B). $\mathrm{TMBr}-3$ consists of 738 nucleotides (including the stop codon) encoding 245 amino acids. Compared with other GenBank sequences it contains a M91T amino acid change. TM5NM1 is 747 base pair long encoding 248 amino acids, carrying an E230G amino acid change. The significance of these alterations is not known. The full coding sequences of mouse TMBr-3 and TM5NM1 were cloned into pET28a plasmid in E. coli and expressed in Luria-Broth medium. The His-tagged proteins were purified from the bacterial pellet using Ni-NTA and hydroxyapatite affinity. The Histag was removed by TEV protease digestion. According to our cloning scheme, the TEV protease cleavage occurred directly before the first methionine of the protein sequence. The average yield of the purified protein was $3-5 \mathrm{mg} / \mathrm{L}$ of E. coli culture. The recombinant TMBr-3 and TM5NM1 proteins produced a band on Coomassie-stained SDSPAGE gel with an apparent molecular mass of approximately 37 and $31 \mathrm{kDa}$, respectively (Fig. 1C). The molecular mass of TMBr-3 and TM5NM1 estimated from the sequence were 28.313 and $28.948 \mathrm{kDa}$, respectively (Protparam). Mass spectrometry gave very similar results to the sequence analysis [28.301 and $28.949 \mathrm{kDa}$ for TMBr-3 and TM5NM1, respectively (data not shown)].

\section{Binding of TMBr-3 and TM5NM1 to Actin Filaments}

To test the ability of recombinant $\mathrm{TMBr}-3$ and TM5NM1 to bind actin filaments (F-actin) cosedimentation assays were performed. Increasing amounts of TMBr-3 or TM5NM1 
were incubated with F-actin $(5 \mu \mathrm{M})$ overnight and the samples were pelleted. The SDS-PAGE analysis revealed that the amount of these tropomyosin isoforms cosedimented with F-actin was increased in a concentration dependent manner, suggesting that both TMBr-3 and TM5NM1 bound to actin

F2 filaments (Fig. 2A). As controls, TMBr-3 and TM5NM1 at different concentrations were also centrifuged in the absence of F-actin. We found that a small amount of tropomyosin appeared in the pellet even without F-actin (data are not shown). The amounts of the pelleted tropomyosin in the absence of actin were quantified at each concentration and used for the correction of the TMBr-3 and TM5NM1 bands obtained with the actin-containing samples. The corrected TM:F-actin band intensity ratios were plotted as a function of tropomyosin concentration (Fig. 2B). Hyperbola fit (Eq. 1) to the data gave dissociation equilibrium constants $\left(K_{\mathrm{d}}\right)$ of $3.5 \pm 0.9 \mu \mathrm{M}$ and $0.1 \pm 0.1 \mu \mathrm{M}$ for the binding of TMBr-3 and TM5NM1 to F-actin, respectively. Although the latter estimate clearly indicates a very tight binding affinity, relatively large errors attributed to the calculated mean. The large error appears probably due to the very tight binding and relatively large applied protein concentrations. To overcome this problem one should decrease the actin

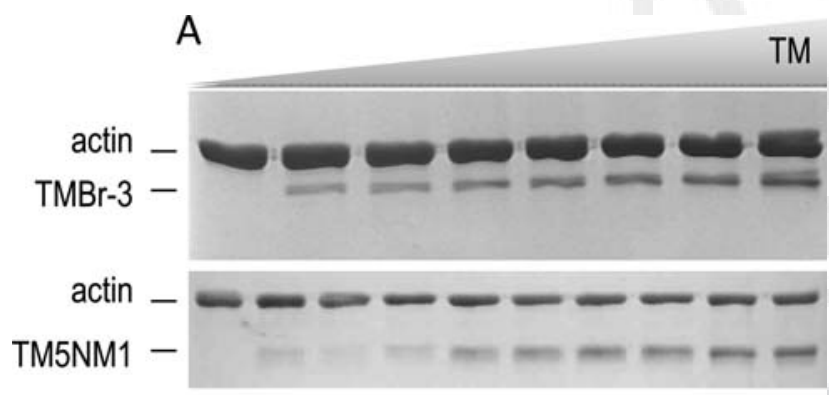

B

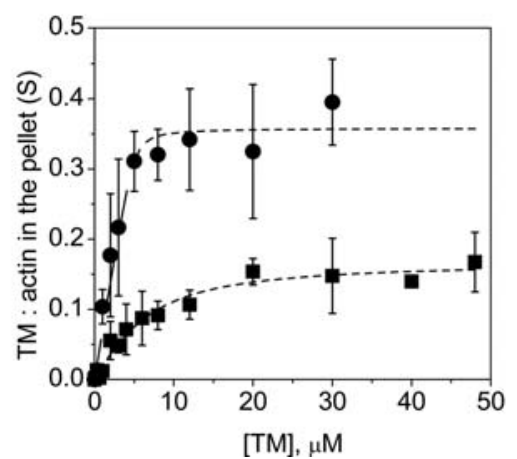

Fig. 2. (A) TMBr-3 (upper panel) or TM5NM1 (lower panel) at different concentrations in the presence of $5 \mu \mathrm{M}$ actin was sedimented, and analyzed on SDS-PAGE by densitometric evaluation. TMBr-3 concentrations are as follows: $0,2,4,6,8,12$, 30 and $40 \mu \mathrm{M}$, for TM5NM1 the concentrations are $0,1,2,3$, 5, 8, 12, 20, $30 \mu \mathrm{M}$. (B) Stoichiometric ratio of tropomyosin to actin in the pellet $(S)$ was plotted as the function of the total tropomyosin concentration (TMBr-3: squares, TM5NM1: circles). Dashed line shows the hyperbola fit to the data using Eq. (1). Data were derived from three independent experiments and the errors are given as standard deviations. concentration in similar experiments. In this work we avoided doing this because they would cause a much larger relative contribution from monomeric actin to the actin pool.

\section{Effects of $\mathrm{TMBr}-3$ and TM5NM1 on Actin Filament Assembly}

Tropomyosins can affect actin filament assembly in an isoform-dependent manner. Muscle tropomyosins, such as recombinant striated muscle and tissue purified rabbit skeletal $\alpha \beta$ TM, slowed down actin polymerization, presumably by their protective effects against the spontaneous mechanical breakage of the actin filaments producing less free ends for the polymerization [Lal and Korn, 1986; Wawro et al., 2007]. In contrast, nonmuscle TM5a and TM2 enhanced actin polymerization by $\sim 30-40 \%$, suggesting a specific effect on barbed end dynamics [Wawro et al., 2007]. To study the effect of TMBr-3 and TM5NM1 on the kinetics of actin assembly, polymerization assays were performed. To quantify our observations the polymerization rates were derived by linear fitting to the $0.45-0.55$ range of the normalized pyrene traces. In agreement with previous observations, we found that $6 \mu \mathrm{M}$ skeletal muscle tropomyosin (skTM) slowed down the assembly of $5 \mu \mathrm{M}$ actin by $\sim 60 \%$ (Fig. 3). Note that considering the affinity of skTM to actin $\left(K_{\mathrm{d}}=0.5 \mu \mathrm{M}\right)$ the $6 \mu \mathrm{M}$ skTM saturated the binding sites on actin in equilibrium. $40 \mu \mathrm{M}$ TMBr-3 and $17 \mu \mathrm{M}$ TM5NM1 (more than 10 -fold greater than the corresponding $K_{\mathrm{d}}$ values) had only minor effect on actin polymerization (Fig. 3). Similarly to skTM, TMBr-3 reduced the rate of actin assembly. While TM5NM1 slightly enhanced actin assembly rate.

\section{Effects of TMBr-3 and TM5NM1 on Actin Filament Disassembly}

Tropomyosins can inhibit the disassembly of actin filaments by their structural stabilization effect [Broschat, 1990]. To

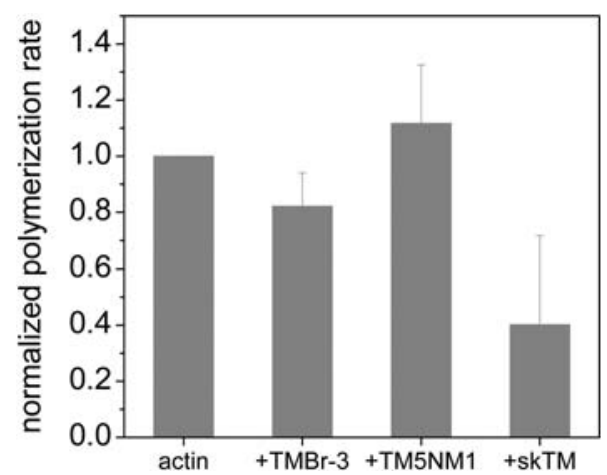

Fig. 3. Normalized rate of actin polymerization $5 \mu \mathrm{M}$ G-actin (5\% pyrene labeled) in the absence and presence of different tropomyosin isoforms, as indicated. The tropomyosin concentrations are as follows; $40 \mu \mathrm{M}$ TMBr-3, $17 \mu \mathrm{M}$ TM5NM1 and $6 \mu \mathrm{M}$ skTM. Data were derived at least from three independent experiments and the errors are given as standard deviations. 
study depolymerization, actin $(2 \mu \mathrm{M}$, containing $50 \%$ pyrene labeled actin) was first polymerized and then diluted into polymerization buffer to reach final concentration of $50 \mathrm{nM}$. This strategy ensures that after dilution the actin concentration is less than the critical concentration, which results in actin filament disassembly dominated by barbed end dynamics. The depolymerization kinetics of actin filaments was followed by monitoring pyrene fluorescence. In the absence of tropomyosin the transients showed single

F4 decreasing phase (Fig. 4A upper panel; black curve). When filaments were preincubated with either $10 \mu \mathrm{M}$ skTM, 40 $\mu \mathrm{M} \mathrm{TMBr}-3$, or $40 \mu \mathrm{M}$ TM5NM1 two phases were observed (Fig. 4A upper panel). The first phase (for approximately $50 \mathrm{~s}$ ) showed slower depolymerization than in the absence of tropomyosin. Then a second, faster phase appeared. In these experiments the buffer to which actin was diluted did not contain tropomyosin. Consequently, after the dilution $(40 \times)$ the tropomyosin concentration has decreased substantially. Due to the alteration of the conditions a new equilibrium was established and most of the tropomyosin dissociated from the filaments. We attribute the first phases of the transients to the depolymerization of actin filaments in the actin-tropomyosin complex, while the second observed phase corresponded to the depolymerization after the dissociation of tropomyosin. To confirm this explanation we repeated the experiments by diluting the actin filaments into buffers containing tropomyosin at the same concentration as in the actin samples $(10 \mu \mathrm{M}$ skTM or $40 \mu \mathrm{M} \mathrm{TMBr}-3)$. The transients obtained under these conditions (Fig. 4A lower panel) were single phased corroborating our conclusions regarding the effect of dissociation of tropomyosin from actin. These observations showed that all the investigated tropomyosins slowed down the depolymerization of actin filaments, and also suggested that dissociation of tropomyosin was slow and occurred on the few tens of seconds time scale. The depolymerization experiments were repeated at various tropomyosin concentrations. The first $50 \mathrm{sec}$ of the transients were fitted with linear functions and the slopes were used to estimate the depolymerization rates. The data showed that these tropomyosin isoforms decreased the depolymerization rate in a concentration dependent manner (Fig. 4B). However, skTM and TM5NM1 had an approximately two-fold greater effect on actin depolymerization than $\mathrm{TMBr}-3$, in correlation with the different affinities of these tropomyosins.

\section{Effects of TMBr-3 and TM5NM1 on the Arp2/3 Complex-Catalyzed Actin Assembly}

Previous biochemical studies revealed that some tropomyosin isoforms inhibit the Arp $2 / 3$ complex-catalyzed actin assembly [Blanchoin et al., 2001; Bugyi et al., 2010]. Cell biological studies revealed that TMBr-3 and TM5NM1 had opposite effect on lamellipodia formation and cell migration [Bryce et al., 2003]. To study the effect of

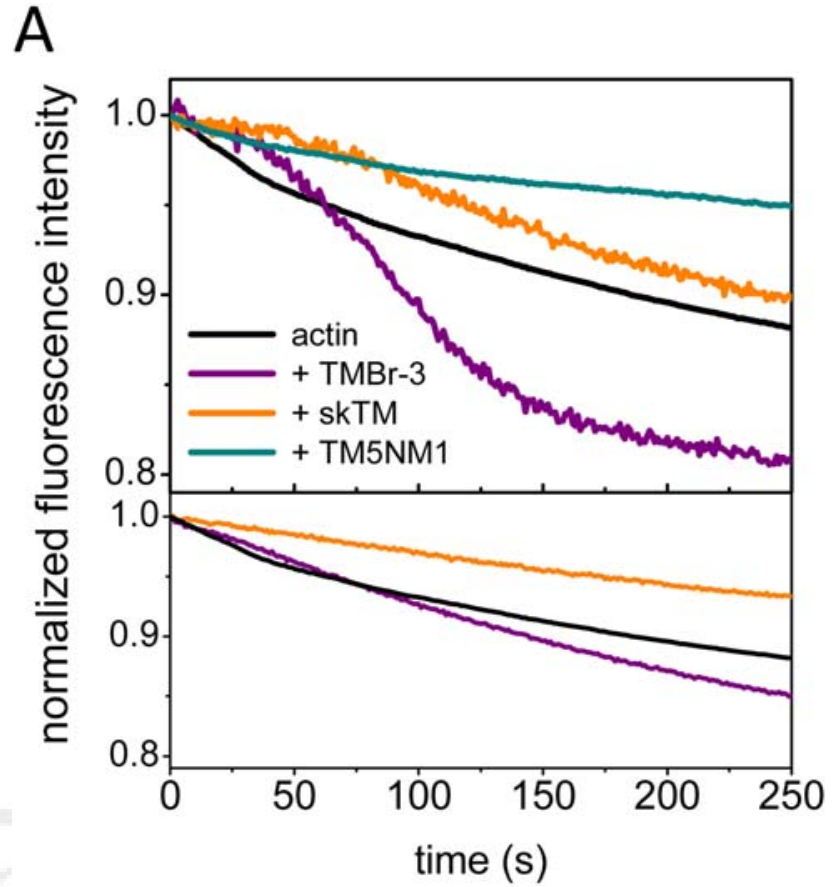

B

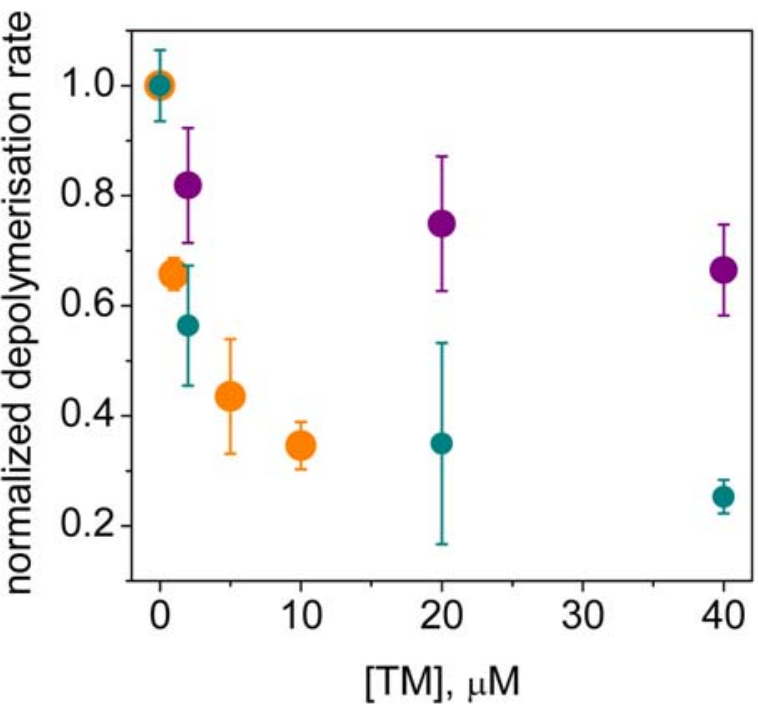
O L

O

Fig. 4. (A) Depolymerization kinetics of $50 \mathrm{nM}$ F-actin in the absence (black line) or presence of $\mathrm{TMBr}-3(40 \mu \mathrm{M}$; purple line), TM5NM1 (40 $\mu \mathrm{M}$; dark cyan line) or skTM $(10 \mu \mathrm{M}$; orange line). The upper panel shows the results obtained when actin was diluted to buffer containing no tropomyosin. The lower panel shows examples of data from experiments in which the dilution was carried out into tropomyosin containing buffer. Fifty nanomolar F-actin in the absence of tropomyosins (black line) or in the presence of $\mathrm{TMBr}-3(40 \mu \mathrm{M}$; purple line) or skTM (10 $\mu \mathrm{M}$; orange line). (B) Normalized depolymerization rates of actin filaments (derived from data similar to those presented in Fig. 4A) as a function of tropomyosin concentration ([TM]). The results are shown for TMBr-3 (purple circles), TM5NM1 (dark cyan circles) and skTM (orange circles). Data were obtained from at least three independent experiments and the errors are given as standard deviations. 
TMBr-3 and TM5NM1 on Arp2/3 complex activity polymerization assays were performed. We found that similarly to skTM, TM5NM1 inhibited the VCA-Arp2/3 complexcatalyzed actin assembly in a concentration-dependent we applied the inhibition was $\sim 25 \%$ (Fig. $5 \mathrm{~B}$ ). Interestingly, we found that TMBr-3 had no effect on Arp $2 / 3$ complex-catalyzed actin assembly (Fig. 5A).

\section{Discussion}

We produced TMBr-3 and TM5NM1 as recombinant proteins in a nontagged form, without any excess amino acids to the native sequence, because any extension at the polypeptide ends may cause unpredictable, even deleterious effects [Bharadwaj et al., 2004]. We successfully purified these tropomyosin isoforms in sufficient amount for in vitro characterization of their interactions with actin. We found that both TMBr-3 and TM5NM1 bind actin filaments, however, their affinities for actin differ (Fig. 2). $\mathrm{TMBr}-3$ binds F-actin with approximately seven-fold lower affinity $\left(K_{\mathrm{d}}=3.48 \pm 0.92 \mu \mathrm{M}\right)$ than $\operatorname{skTM}\left(K_{\mathrm{d}}=0.5 \mu \mathrm{M}\right)$
[Boussouf et al., 2007], while TM5NM1 has somewhat tighter affinity $\left(K_{\mathrm{d}}=0.10 \pm 0.16 \mu \mathrm{M}\right)$ than skTM. These differences in the binding strength are consistent with previous results, in showing that the affinity of tropomyosins to actin strongly depends on the specific combination of exons encoding the $\mathrm{N}$-, and $\mathrm{C}$-terminus of tropomyosins [Moraczewska et al., 1999]. For the tropomyosin isoforms studied in this paper, the 1b1d exon pair (Fig. 1A) is expected to endow the TM5NM1 isoform with the highest affinity, exons 1a9a with acetylation (for tissue purified skTM) results in somewhat lower affinity, while the $1 b 9 c$ exon combination in $\mathrm{TMBr}-3$ results in poor binding to actin filaments. Our results support the view that the $\mathrm{N}$ - and C-termini of tropomyosins are important determinants of actin affinity by establishing the ability of tropomyosin isoforms to form end-to-end polymers along the actin filament. The lower affinity of $\mathrm{TMBr}-3$ binding to actin filaments is also consistent with previous findings in showing that brain isoforms isolated from tissue bind actin with approximately 10 -fold lower affinity than muscle isoforms [Broschat and Burgess, 1986]. It would also explain the previous results showing that saturation of the actin
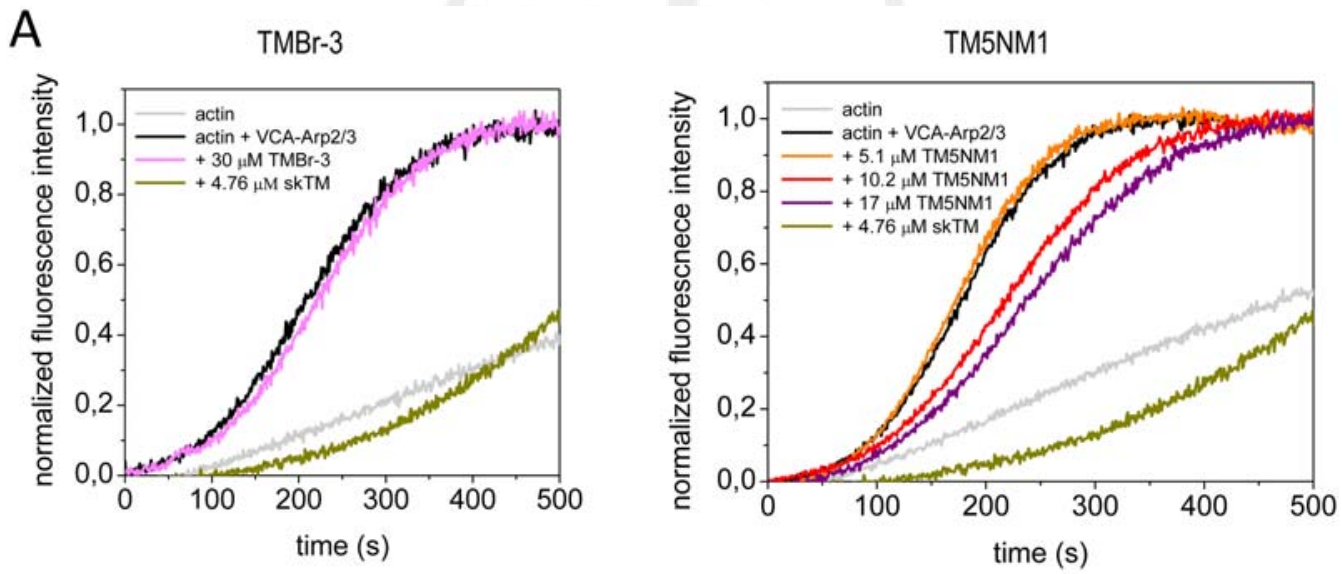

$\mathrm{C}$

$\mathrm{O}$

$\mathrm{L}$

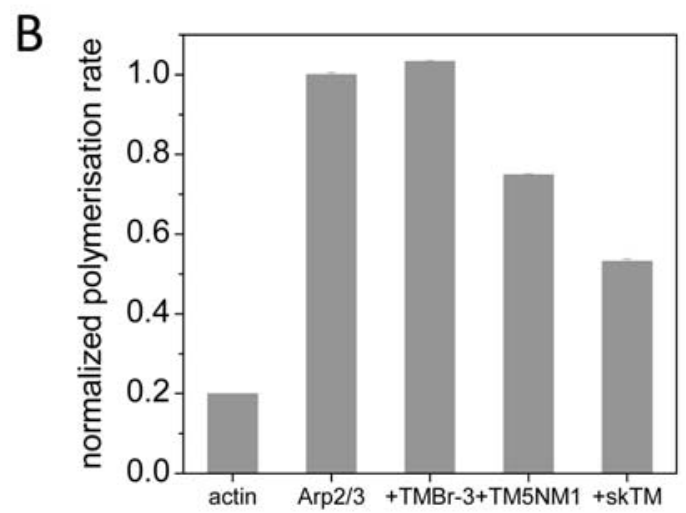

Fig. 5. (A) Time courses of VCA-Arp $2 / 3$ complex catalyzed actin assembly monitored by the change in pyrene fluorescence in the absence (black line) and presence of different tropomyosin isoforms, as indicated. Left panel: the TMBr- 3 concentration is $30 \mu \mathrm{M}$ (magenta line). Right panel: the TM5NM1 concentrations are 5.1 $\mu \mathrm{M}$ (orange line), $10.2 \mu \mathrm{M}$ (red line) and $17 \mu \mathrm{M}$ (purple line). The skTM concentration is $4.76 \mu \mathrm{M}$ (dark yellow line). [G-actin] $=2.5 \mu \mathrm{M}$ (5\% pyrene labeled), [Arp2/3 complex] $=100 \mathrm{nM}$, $[\mathrm{VCA}]=200 \mathrm{nM}$. Light gray curve shows the kinetics of spontaneous actin assembly. (B) Normalized rate of VCA-Arp $2 / 3$ complex catalyzed actin polymerization (derived from data similar to those presented in Fig. 5A) in the absence and presence of different tropomyosin isoforms, as indicated. The tropomyosin concentrations are as follows; $40 \mu \mathrm{M}$ TMBr-3, $17 \mu \mathrm{M}$ TM5NM1, and $4.76 \mu \mathrm{M}$ skTM. 
filaments by TMBr-3 cannot be reached by adding up to $10 \mu \mathrm{M}$ of tropomyosin without troponin or myosin [Moraczewska et al., 1999]. It should be also considered that in nonmuscle cells tropomyosins associate exclusively with nonmuscle $\beta$ and $\gamma$ actin isoforms, rather than the muscle specific $\alpha$ isoform that was used in our study [Weinberger et al., 1996]. Some isoform-dependent features can establish specific actin and tropomyosin pairs with higher binding affinity and preferential binding for optimal functioning. As example, isoform-specific interactions were already shown for profilin and formin isoforms [Neidt et al., 2009]. The interaction of many actin-binding proteins with monomeric or filamentous actin can be antagonistically or synergistically altered by the presence of other proteins. As a relevant example, caldesmon-a calcium/ calmodulin regulated actin-binding protein-is known to enhance the binding of low molecular weight tropomyosins to actin [Yamashiro-Matsumura and Matsumura, 1988]. Since caldesmon is expressed in brain [Ueki et al., 1987], we speculate that it may also promote the association of the $\mathrm{TMBr}-3$ isoforms to actin filaments.

SkTM at saturating amount slows down both actin assembly and disassembly, by reducing the association and dissociation rate constants of actin monomers to and from the filaments (Figs. 3 and 4) [Lal and Korn, 1986; Broschat, 1990]. We found that TMBr-3 had a similar but smaller effects on the rate of actin polymerization and depolymerization (Figs. 3 and 4). This observation may be explained by assuming that $\mathrm{TMBr}-3$ decreases the rate constant for the association of actin monomers to the ends of the filaments to a lower extent than skTM. To corroborate this assumption in cosedimentation assays we found that the amount of actin in the supernatants were independent of the TMBr-3 concentration (data not shown). Accordingly, under polymerizing conditions the concentration of the monomeric actin was the same in the absence and presence of $\mathrm{TMBr}-3$, indicating that TMBr-3 did not change the critical concentration. Based on the unchanged critical concentration one would expect that $\mathrm{TMBr}-3$ has the same magnitude of effect on the association and dissociation rates of the actin monomers. It was thus expected that TMBr-3 has the same effect on the association rate constant, which correlates with the slight inhibition of actin assembly by $\mathrm{TMBr}-3$ (Fig. 3). In depolymerization experiments we found evidence that the dissociation of the tropomyosin from actin is slow, occurring within 50-100 sec. Thus, the corresponding micromolar dissociation equilibrium constant of the TM-actin complex suggest that tropomyosin association to the filaments must also be slow falling into the $10^{4} \mathrm{M}^{-1} \mathrm{~s}^{-1}$ range. In this case the binding of tropomyosin would occur on a second time-scale under our experimental conditions, which is comparable with the rate of actin filament growth. Therefore, in these measurements we did not observe the full effect of TMBr-3 on the polymerization rate. In the depolymerization experiments actin was preincubated with tropomyosin and the equilibrium has been reached prior to the measurements. These considerations can explain the small deviation of the experimental data from the expectations based on the unchanged critical concentration. Our data also implicated that TMBr-3 association to actin filaments is approximately 60-fold slower than that of actin monomers. We found that TM5NM1 slightly stimulated actin assembly, while inhibited actin disassembly at the same extent as skTM (Figs. 3 and 4). In previous studies TM5a and TM2 isoforms behaved similarly to TM5NM1, by increasing the polymerization rate and inhibiting the depolymerization rate [Kostyukova and Hitchcock-DeGregori, 2004; Wawro et al., 2007]. The Cterminus of tropomyosin points toward the barbed end of the actin filament, and this part is common for TM5NM1, TM5a, and TM2 (exon 9d) but differ for skTM and TMBr-3 (exon 9a or 9c, respectively) (Fig. 1A). While the $\mathrm{N}$-terminus facing toward the pointed end is the same for skTM and TM2 (exons 1a2b) or TM5a, TMBr-3, and TM5NM1 (exon 1b) (Fig. 1A). Although sequence and hence structure-specific effects of the individual tropomyosin termini can be assumed to have impact on actin filament end dynamics, no simple correlations seem to exist. Considering the magnitude of the effects of different tropomyosins (TM5a/TM5NM1 $>$ skTM $>$ TM2 $>$ TMBr-3) this raises the possibility of the importance of actin affinity, which in turn is determined by the head-to-tail interactions of the tropomyosin ends [Moraczewska et al., 1999]. In that respect the C-terminal exon 9c of $\mathrm{TMBr}-3$ (shared with $\mathrm{TMBr}-1$ isoform) may have a significant concern on the observed low binding strength and the moderate effects on actin dynamics.

Similarly to all the previously studied tropomyosin isoforms (rabbit skTM, chicken striated muscle TM, TM5a, and TM2) [Blanchoin et al., 2001; Bugyi, 2010], TM5NM1 inhibited the VCA-Arp2/3 complex catalyzed actin assembly (Fig. 5). In contrast, we found that $\mathrm{TMBr}-3$ did not have any effect of the nucleation catalyzing activity of the VCAArp2/3 complex machinery (Fig. 5). This is a unique feature of $\mathrm{TMBr}-3$, since so far this is the only tropomyosin isoform, which allows Arp2/3 complex-dependent actin assembly. The different effects of TMBr-3 and TM5NM1 on Arp2/3 complex-catalyzed actin assembly is in good agreement with the observations from cellular studies. In B35 neuroepithelial cells the expression of TMBr-3 or TM5NM1 cells resulted in opposite effects on the leading edge region and lamellipodia formation; excess TMBr-3 induced lamellipodia formation, while TM5NM1 decreased lamellipodia [Bryce et al., 2003]. Also, in the brain the Arp $2 / 3$ complex machinery is involved in the cytoskeletal organization of both post- and presynaptic actin structures that can be also associated with TMBr-3 [Had et al., 1994; Hotulainen et al., 2009; Hotulainen and Hoogenraad, 2010; Spillane et al., 2011]. The inhibitory effect of tropomyosins on Arp2/3 complex activity is not attributed to differences in the ATP hydrolysis and 
phosphate release rate or debranching of daughter filaments, it rather results from a competitive binding of the two proteins to actin filaments [Blanchoin et al., 2001; Bugyi, 2010]. The exact molecular origin of the competitive binding between tropomyosin and Arp2/3 complex is not known. Structural modeling revealed that the actin filament binding sites of skTM and Arp2/3 complex partially overlap [Rouiller et al., 2008], suggesting that the functional inhibition results from sterical hindrance. No structural data are available for TMBr-3 and TM5NM1 yet. However, previous studies showed that the azimuthal equilibrium position of the tropomyosins around the actin filament can be isoform-specific [Lehman et al., 2000]. Thus, the different effects of tropoymosins on the activity of the Arp $2 / 3$ complex can originate from the different positions of the different tropomyosin isoforms on actin filaments. This assumes that TMBr-3 occupies positions on the actin filament different from those tropomyosins that inhibit the activity of the Arp2/3 complex. On the other hand, it cannot be excluded that differences in the structural and kinetic features of the head-to-tail complex formation, which is an important determinant of actin filament binding, can cause the differences in the effects of tropomyosins on the interaction of actin with the Arp $2 / 3$ complex machinery. In conclusion, we propose that distinct tropomyosin isoforms can have different kinetic and structural features and flexibility to occupy and maintain a characteristic localization on the actin filament. This isoform dependent nature of the tropomyosin-actin interactions is also important in the competition for shared binding sites with other actin-binding proteins, such as the Arp2/3 complex, which plays essential roles in the manifestation and regulation of the biological functions of tropomyosins.

Although more information will be required to understand the exact roles of these tropomyosins in nonmuscle cells, the current observations suggest that their functions differ substantially. In future studies it would also be important to investigate whether the presence of other actinregulatory proteins, such as caldesmon, $\mathrm{ADF} /$ cofilins and myosins and $\beta$ and $\gamma$ actin isoforms have an impact on the interaction of the TMBr-3 and TM5NM1 with actin.

\section{Materials and Methods}

\section{Molecular Cloning and Protein Preparation}

The mouse TMBr-3 tropomyosin construct was generated from a previously cloned sequence in pET28a plasmids containing an N-terminal FXa protease recognition site. TM5NM1 was cloned de novo from total mRNA starting with reverse transcription and PCR to produce cDNA. We designed target-specific PCR primers: the forward primer was 5'- TAT TTT CAG ATG GCG GGG AGT AGC TCG CT $-3^{\prime}$ and the reverse primer was $5^{\prime}$ - TCT AAG CTT TTA ATC CTC ATT CAG GGC CA $-3^{\prime}$ for TMBr-3, and 5'- TAT TTT CAG atg gcc ggg acc acc acc atc $-3^{\prime}$ and $5^{\prime}-$ TCT AAG CTT TTA CAT CTC GTT CAG GT $-3^{\prime}$ for TM5NM1. The introduction of a HindIII restriction endonuclease site is underlined. The thermal cycler conditions were as follows: $95^{\circ} \mathrm{C}$ for $5 \mathrm{~min}, 38$ cycles of $95^{\circ} \mathrm{C}$ for $30 \mathrm{~s}$ denaturation, $50^{\circ} \mathrm{C}$ for $1 \mathrm{~min}$ annealing, and $72^{\circ} \mathrm{C}$ for $1 \mathrm{~min}$ synthesis, then $72^{\circ} \mathrm{C}$ for $5 \mathrm{~min}$. For TM5NM1 we applied 10 cycles at $42^{\circ} \mathrm{C}$ for $1 \mathrm{~min}$ and 30 cycles at $51^{\circ} \mathrm{C}$ for $1 \mathrm{~min}$ as annealing. In a second round PCR an EcoRI restriction endonuclease (underlined) and a ProTEV protease site (double underlined) were tailored Nterminal to the coding sequence using the 5'- CTC GAA TTC GAA AAC CTG TAT TTT CAG ATG $-3^{\prime}$ forward primer and the above reverse primers. The thermal cycler conditions were: $95^{\circ} \mathrm{C}$ for $5 \mathrm{~min}, 40$ cycles of $95^{\circ} \mathrm{C}$ for 30 sec denaturation, $58^{\circ} \mathrm{C}$ for $1 \mathrm{~min}$ annealing, and $72^{\circ} \mathrm{C}$ for 1 min synthesis, then $72^{\circ} \mathrm{C}$ for $5 \mathrm{~min}$. The PCR product was cloned into 6xHis/pET28a expression plasmid (NovaGen) between the EcoRI and HindIII restriction sites. The recombinant protein was expressed in E. coli BL21 (DE3) cells using $30 \mu \mathrm{g} / \mathrm{ml}$ kanamycin as selection antibiotics. A preculture in $5 \mathrm{ml}$ was prepared over night at $37^{\circ} \mathrm{C}$, and used to inoculate $3 \mathrm{~L}$ Luria Broth medium. The cells were grown to $\mathrm{OD}_{600} 0.6-0.8$ at $37^{\circ} \mathrm{C}$ and induced by $1 \mathrm{mM}$ IPTG (Sigma) for $4 \mathrm{~h}$. Bacterial pellet was resuspended in lysis buffer $\left(50 \mathrm{mM} \mathrm{NaH} \mathrm{PO}_{4}\right.$ buffer $\mathrm{pH} 8.0,500 \mathrm{mM}$ $\mathrm{NaCl}$ ), containing $2 \mu \mathrm{g} / \mathrm{ml}$ DNase, $5 \mathrm{mM} \mathrm{MgCl}_{2}, 1 \times$ complete protease inhibitor mix (Sigma), and $0.2 \mathrm{mM}$ PMSF, lyzed by five cycles of freeze-thaw and sonication $(5 \times 1 \mathrm{~min})$, then centrifuged $(6000 \times g$ for $15 \mathrm{~min}$ at $4^{\circ} \mathrm{C}$, Sorvall, T-1250 rotor). The supernatant was loaded to Ni-NTA chelating column (QIAGEN) equilibrated with lysis buffer, and eluted with 5, 10, 20, 40, and $250 \mathrm{mM}$ imidazole (Sigma) in lysis buffer. The peak fractions were pooled and dialyzed against hydroxyapatite column buffer (1 mM Na-phosphate buffer pH 7.0, $1 \mathrm{M} \mathrm{KCl}, 2.5 \mathrm{mM}$ DTT). The sample was loaded on a hydroxyapatite CHT column (Bio-Rad), and eluted with $300 \mathrm{mM} \mathrm{Na-phosphate}$ buffer, $\mathrm{pH}$ 7.0, $1 \mathrm{M} \mathrm{KCl}$ and 2.5 mM DTT. The peak fractions were pooled and dialyzed against storage buffer (5 mM Tris pH 7.5, $100 \mathrm{mM} \mathrm{KCl,} 1 \mathrm{mM} \mathrm{DTT}$ ). The Histag was cleaved by TEV protease at $37^{\circ} \mathrm{C}$, adjusting the amount of protease and the time of incubation empirically. The digested protein was again purified on a Ni-NTA column to remove the His-tag and the TEV protease. The flowthrough was concentrated, ultracentrifuged $(676,000$ $\times g$ for 20 min at $4^{\circ} \mathrm{C}$ Beckman-Coulter, MLA-130 rotor), and the concentration was measured using a BCA kit (Sigma). Protein preparations were stored at $0^{\circ} \mathrm{C}$ in $5 \mathrm{mM}$ Tris $\mathrm{pH}$ 7.5, $100 \mathrm{mM} \mathrm{KCl}$, and $1 \mathrm{mM}$ DTT.

TEV protease was purified using a $6 \times$ His-tag pET24 (TEV-235) plasmid construction (a kind gift of Hüseyin Besir EMBL) in E.coli BL21 DE3 cells. Freshly transformed bacteria were plated on LB-agar containing $30 \mu \mathrm{g} / \mathrm{ml}$ kanamycin, and $60 \mathrm{ml}$ preculture was grown over night at $37^{\circ} \mathrm{C}$. It was then used in $2 \%$ to inoculate $31 \mathrm{LB}$ medium 
grown to $\mathrm{OD}_{600} 0.6-0.8$ at $37^{\circ} \mathrm{C}$. The temperature was reduced to $30^{\circ} \mathrm{C}$ for $45 \mathrm{~min}$, and expression was induced by adding $1 \mathrm{mM}$ IPTG for $4 \mathrm{~h}$ at $30^{\circ} \mathrm{C}$. Bacterial pellet was resuspended in lysis buffer $(50 \mathrm{mM} \mathrm{Na}$-phosphate buffer $\mathrm{pH}$ 8.0, $100 \mathrm{mM} \mathrm{NaCl}, 25 \mathrm{mM}$ imidazole, $10 \%$ glycerol) containing $2 \mu \mathrm{g} / \mathrm{ml}$ DNase, $5 \mathrm{mM} \mathrm{MgCl}_{2}, 1 \times$ complete protease inhibitor mix (Sigma), $0.2 \mathrm{mM}$ PMSF and $500 \mu \mathrm{g} / \mathrm{ml}$ lysozyme. It was incubated for $10 \mathrm{~min}$ at RT and cells were further lyzed by sonication $(6 \times 25 \mathrm{sec})$. The sample was centrifuged $\left(100,000 \times g\right.$ for $1 \mathrm{~h}$ at $4^{\circ} \mathrm{C}$, Sorvall, T-1250 rotor), and the supernatant was loaded on Ni-NTA column equilibrated with lysis buffer. The column was incubated at $4^{\circ} \mathrm{C}$ for $2 \mathrm{~h}$ then washed with $15 \mathrm{ml}$ lysis buffer and $10 \mathrm{ml}$ elution buffer $(50 \mathrm{mM} \mathrm{Na}$-phosphate buffer $\mathrm{pH} 8.0,100 \mathrm{mM} \mathrm{NaCl}, 500 \mathrm{mM}$ imidazole, $10 \%$ glycerol). The latter fraction was dialyzed against IEX running buffer (50 mM Na-phosphate buffer pH 8.0, 100 $\mathrm{mM} \mathrm{NaCl}, 1 \mathrm{mM}$ EDTA, $1 \mathrm{mM}$ DTT, 10\% glycerol), and further purified on anion exchange source $15 \mathrm{Q}$ column with IEX elution buffer (50 mM Na-phosphate buffer $\mathrm{pH}$ 8.0, $1 \mathrm{M} \mathrm{NaCl}, 1 \mathrm{mM}$ EDTA, $1 \mathrm{mM}$ DTT, 10\% glycerol). The peak fractions were pooled and checked on SDSPAGE and ultracentrifuged $(676,000 \times g$ for $20 \mathrm{~min}$ at $4^{\circ} \mathrm{C}$, Beckman-Coulter, MLA-130 rotor). The protein concentration was determined photometrically using $\varepsilon_{280}=1.19$ $\mathrm{ml}^{*} \mathrm{mg}^{-1 *} \mathrm{~cm}^{-1}$. The final glycerol content was adjusted to $50 \%$ and the samples were stored at $-20^{\circ} \mathrm{C}$.

Actin was prepared from rabbit hind leg muscle [Spudich and Watt, 1971] and gel filtered in a Superdex G75 (GE Healthcare) column in buffer A ( 4 mM Tris $\mathrm{pH} 7.5,0.2$ mM ATP, $0.1 \mathrm{mM} \mathrm{CaCl}_{2}, 0.5 \mathrm{mM} \mathrm{MEA,} \mathrm{0.005 \%} \mathrm{NaN}_{3}$ ). $\mathrm{G}$-actin was stored on ice in buffer $\mathrm{A}$ until used. Before polymerization $\mathrm{CaCl}_{2}$ was exchanged for $\mathrm{MgCl}_{2}$ with EGTA.

Arp2/3 complex from bovine brain and recombinant GST-tagged human VCA were purified as described previously [Egile et al., 1999].

skTM was purified from the remnants of the actin acetone powder [Smillie, 1982], then applied to hydroxyapatite chromatography, and stored frozen in $5 \mathrm{mM}$ Tris $\mathrm{pH} 7.8$ and $1 \mathrm{mM}$ DTT. For the fluorescent measurements actin was labeled with pyrenyl-iodoacetamide as described previously (pyrene, Invitrogen) [Kouyama and Mihashi, 1981]. The protein concentrations were measured photometrically using $\varepsilon_{280}=1.11 \mathrm{ml}^{*} \mathrm{mg}^{-1 *} \mathrm{~cm}^{-1}$ and $\varepsilon_{290}=0.63 \mathrm{ml}^{*} \mathrm{mg}^{-1 *} \mathrm{~cm}^{-1}$ extinction coefficients for actin and $\varepsilon_{280}=0.3 \mathrm{ml}^{*} \mathrm{mg}^{-1 *} \mathrm{~cm}^{-1}$ for skTM.

\section{Co-Sedimentation Assays}

Twenty micromolar Mg-G-actin in buffer A was polymerized with $2 \mathrm{mM} \mathrm{MgCl}_{2}$ and $100 \mathrm{mM} \mathrm{KCl}$, then the F-actin solution was diluted to $5 \mu \mathrm{M}$ in the absence or presence of different concentrations of tropomyosin in polymerization buffer (buffer A supplemented with $2 \mathrm{mM} \mathrm{MgCl}_{2}$ and $100 \mathrm{mM}$
$\mathrm{KCl}$ (final concentrations)). Samples $(60 \mu \mathrm{l})$ were incubated over night at $4^{\circ} \mathrm{C}$, then pelleted $(440,000 \times g$ for $30 \mathrm{~min}$ at $4^{\circ} \mathrm{C}$, Beckman-Coulter, TLA-100 rotor). Pellets and supernatants were separated and analyzed by SDS-PAGE [Laemmli, 1970]. The protein bands on the gel were quantified using densitometry (GeneTools) after Coomassie staining. Control experiments showed that Coomassie stains actin and these tropomyosin isoforms equally (data not shown). The TM : actin ratio in the pellet was calculated and plotted as a function of tropomyosin concentration. The data were fit using the following equation:

$$
\begin{aligned}
S= & \frac{1}{2} *\left[\left([T M]_{0}+[A]_{0}+K_{d}\right) * \frac{S_{\max }}{[A]_{0}}\right. \\
& -\sqrt{\left(\left([T M]_{0}+[A]_{0}+K_{d}\right) * \frac{S_{\max }}{[A]_{0}}\right)^{2}-4 * \frac{S_{\max }^{2}}{[A]_{0}}} \\
& \left.\left.*[T M]_{0}\right]\right]
\end{aligned}
$$

where $S$ is the TM: actin ratio in the pellet, $S_{\max }$ is the maximum value of $S$ measured at saturating TM concentration, $[T M]_{0}$ and $[A]_{0}$ are the total concentration of TM and actin in the samples, respectively and $K_{\mathrm{d}}$ is the dissociation equilibrium constant of the TM-F-actin complex.

\section{Fluorescence Measurements}

Fluorescence experiments were performed using a PerkinElmer or a Jobin Yvon spectrofluorimeter (Horiba Scientific). In all the experiments the actin-bound calcium was replaced with magnesium by adding $0.2 \mathrm{mM}$ EGTA and $0.05 \mathrm{mM} \mathrm{MgCl}_{2}$ (final concentrations) and incubating the samples for 5-10 min at room temperature. Pyrene fluorescence was excited and the emission was detected at $365 \mathrm{~nm}$ and $407 \mathrm{~nm}$, respectively. Measurements were carried out at $22^{\circ} \mathrm{C}$. When concentration dependence was measured the total volume of tropomyosin and its storing buffer was always kept constant and represented $40 \%$ of the total volume of the sample.

\section{Polymerization Assays.}

Five micromolar $\mathrm{Mg}-\mathrm{G}$-actin in buffer A (containing 5\% pyrene labeled actin) was polymerized in the presence of $2 \mathrm{mM} \mathrm{MgCl}_{2}, 100 \mathrm{mM} \mathrm{KCl}$ (final concentrations) and different amounts of tropomyosin. Polymerization kinetics was followed by measuring the changes in pyrene fluorescence in time. The fluorescence transients were normalized and the polymerization rates were determined by fitting the linear part $(0.45-0.55)$ of the data. Average rates from at least three independent measurements were calculated.

\section{Depolymerization Assays.}

Ten micromolar Mg-G-actin in buffer A (containing 50\% pyrene labeled actin) was polymerized over night by adding 
$2 \mathrm{mM} \mathrm{MgCl} 2$ and $100 \mathrm{mM} \mathrm{KCl}$ (final concentrations) in the absence or presence of tropomyosin. The F-actin solution was diluted to $50 \mathrm{nM}$ into polymerization buffer. Depolymerization kinetics was followed by measuring the decrease in pyrene fluorescence in time. Depolymerization rates were estimated by linear fitting the first $50 \mathrm{sec}$ of the pyrene transients. Average rates from at least three independent measurements were calculated. Normalized depolymerization rates were calculated as the ratio of the depolymerization rate in the absence of tropomyosin to the depolymerization rate in the presence of tropomyosin.

\section{Acknowledgments}

This study was supported by a grant from the Hungarian Science Foundation (OTKA grants K77840 (MN), NN10776 (MN) and PD 83648 (BB), K109689 (BB)) and grants from the Hungarian National Office for Research and Technology (GVOP grants GVOP-3.2.1.-2004-04-0190/3.0 and GVOP-3.2.1.-2004-04-0228/3.0 (MN)). This work was also supported by 'Science, Please! Research Team on Innovation' (SROP-4.2.2/08/1/2008-0011), by University of Pécs, Medical School (KA No: 2011/34039/OTKA2011-8) and by National Innovation Office "Baross Gábor" Programme (REG-DD-09-1-2009-0009 Tirfm_09). BB is a Bolyai Fellow of the Hungarian Academy of Sciences. We wish to thank Éva Hoffmann for her skills and contribution to the protein preparations. We thank Tamás Huber for his help in TEV protease expression and purification. We highly appreciate the help from László Márk (Department of Biochemistry and Medical Chemistry, University of Pécs) who carried out the mass spectrometry analysis.

\section{References}

Bharadwaj S, et al. 2004. N terminus is essential for tropomyosin functions: N-terminal modification disrupts stress fiber organization and abolishes anti-oncogenic effects of tropomyosin-1. J Biol Chem AQ3 AQ4 279(14):14039-14048.

Blanchoin L, Pollard TD, Hitchcock-DeGregori SE. 2001. Inhibition of the Arp2/3 complex-nucleated actin polymerization and branch formation by tropomyosin. Curr Biol 11(16):13001304 .

Boussouf SE, et al. 2007. Role of tropomyosin isoforms in the calcium sensitivity of striated muscle thin filaments. J Muscle Res Cell Motil 28(1):49-58.

Broschat KO. 1990. Tropomyosin prevents depolymerization of actin filaments from the pointed end. J Biol Chem 265(34):2132321329.

Broschat KO, Burgess DR. 1986. Low Mr tropomyosin isoforms from chicken brain and intestinal epithelium have distinct actinbinding properties. J Biol Chem 261(28):13350-13359.

Bryce NS, et al. 2003. Specification of actin filament function and molecular composition by tropomyosin isoforms. Mol Biol Cell 14(3):1002-1016.

Bugyi B, Didry D, Carlier MF. 2010. How tropomyosin regulates lamellipodial actin-based motility: a combined biochemical and reconstituted motility approach. Embo J 29(1):14-26.
Coulton AT, et al. 2008. Role of the head-to-tail overlap region in smooth and skeletal muscle beta-tropomyosin. Biochemistry $47(1)$ : 388-397.

Egile C, et al. 1999. Activation of the CDC42 effector N-WASP by the Shigella flexneri IcsA protein promotes actin nucleation by Arp $2 / 3$ complex and bacterial actin-based motility. J Cell Biol 146(6):1319-1332.

Fine RE, et al. 1973. Tropomyosin in brain and growing neurones. Nat New Biol 245(145):182-186.

Flicker PF, Phillips GN., Jr., Cohen C. 1982. Troponin and its interactions with tropomyosin. An electron microscope study. J Mol Biol 162(2):495-501.

Gunning P, et al. 1998. Isoform sorting and the creation of intracellular compartments. Annu Rev Cell Dev Biol 14:339-372.

Gunning PW, et al. 2005. Tropomyosin isoforms: divining rods for actin cytoskeleton function. Trends Cell Biol 15(6):333-341.

Gunning P, O’Neill G, Hardeman E. 2008. Tropomyosin-based regulation of the actin cytoskeleton in time and space. Physiol Rev 88(1):1-35.

Had L, et al. 1994. Tropomyosin isoforms in rat neurons: the different developmental profiles and distributions of TM- 4 and TMBr-3 are consistent with different functions. J Cell Sci 107 (Pt 10):2961-2973.

Hannan AJ, et al. 1995. Intracellular localization of tropomyosin mRNA and protein is associated with development of neuronal polarity. Mol Cell Neurosci 6(5):397-412.

Hotulainen P, Hoogenraad CC. 2010. Actin in dendritic spines: connecting dynamics to function. J Cell Biol 189(4):619-629.

Hotulainen P, et al. 2009. Defining mechanisms of actin polymerization and depolymerization during dendritic spine morphogenesis. J Cell Biol 185(2):323-339.

Kostyukova AS, Hitchcock-DeGregori SE. 2004. Effect of the structure of the $\mathrm{N}$ terminus of tropomyosin on tropomodulin function. J Biol Chem 279(7):5066-5071.

Kouyama T, Mihashi K. 1981. Fluorimetry study of N-(1-pyrenyl)iodoacetamide-labelled F-actin. Local structural change of actin protomer both on polymerization and on binding of heavy meromyosin. Eur J Biochem 114(1):33-38.

Laemmli UK. 1970. Cleavage of structural proteins during the assembly of the head of bacteriophage T4. Nature, 227(5259):680-685.

Lal AA, Korn ED. 1986. Effect of muscle tropomyosin on the kinetics of polymerization of muscle actin. Biochemistry 25(5): 1154-1158.

Lees-Miller JP, Goodwin LO, Helfman DM. 1990. Three novel brain tropomyosin isoforms are expressed from the rat alphatropomyosin gene through the use of alternative promoters and alternative RNA processing. Mol Cell Biol 10(4):1729-1742.

Lehman W, et al. 2000. Tropomyosin and actin isoforms modulate the localization of tropomyosin strands on actin filaments. J Mol Biol 302(3):593-606.

Moraczewska J, Nicholson-Flynn K, Hitchcock-DeGregori SE. 1999. The ends of tropomyosin are major determinants of actin affinity and myosin subfragment 1 -induced binding to F-actin in the open state. Biochemistry 38(48):15885-15892.

Neidt EM, Scott BJ, Kovar DR. 2009. Formin differentially utilizes profilin isoforms to rapidly assemble actin filaments. J Biol Chem 284(1):673-684.

Perrin BJ, Ervasti JM. 2010. The actin gene family: function follows isoform. Cytoskeleton (Hoboken) 67(10):630-634.

Rouiller I, et al. 2008. The structural basis of actin filament branching by the Arp2/3 complex. J Cell Biol 180(5):887-895. 
Schevzov G, et al. 1997. Tropomyosin localization reveals distinct populations of microfilaments in neurites and growth cones. Mol Cell Neurosci 8(6):439-454.

Schevzov G, et al. 2005. Tissue-specific tropomyosin isoform composition. J Histochem Cytochem 53(5):557-570.

Smillie LB. 1982. Preparation and identification of alpha- and betatropomyosins. Methods Enzymol 85(Pt B):234-241.

Spillane M, et al. 2011. The actin nucleating Arp2/3 complex contributes to the formation of axonal filopodia and branches through the regulation of actin patch precursors to filopodia. Dev Neurobiol 71(9):747-758.

Spudich JA, Watt S. 1971. The regulation of rabbit skeletal muscle contraction. I. Biochemical studies of the interaction of the tropomyosin-troponin complex with actin and the proteolytic fragments of myosin. J Biol Chem 246(15):4866-4871.

Stamm S, et al. 1993. Brain-specific tropomyosins TMBr-1 and TMBr-3 have distinct patterns of expression during development and in adult brain. Proc Natl Acad Sci USA 90(21):9857-9861.
Ueki N, et al. 1987. Expression of high and low molecular weight caldesmons during phenotypic modulation of smooth muscle cells. Proc Natl Acad Sci USA 84(24):9049-9053.

Vindin H, Gunning P. Cytoskeletal tropomyosins: choreographers of actin filament functional diversity. J Muscle Res Cell Motil. (in press).

Wawro B, et al. 2007. Tropomyosin regulates elongation by formin at the fast-growing end of the actin filament. Biochemistry 46(27): 8146-8155.

Weinberger RP, et al. 1993. Induction of neuron-specific tropomyosin mRNAs by nerve growth factor is dependent on morphological differentiation. J Cell Biol 120(1):205-215.

Weinberger R, et al. 1996. The molecular composition of neuronal microfilaments is spatially and temporally regulated. J Neurosci 16(1):238-252.

Yamashiro-Matsumura S, Matsumura F. 1988. Characterization of 83kilodalton nonmuscle caldesmon from cultured rat cells: stimulation of actin binding of nonmuscle tropomyosin and periodic localization along microfilaments like tropomyosin. J Cell Biol 106(6):1973-1983. 
AQ1: Please check whether affiliation 1 is OK as typeset.

AQ2: Please provide department/division name for affiliations 2, 3.

AQ3: Please provide complete author names for all et al. type reference.

AQ4: Please note that journal style for references is [Name, Date] in text citations and in alphabetical order in the reference section. This has been applied for the proof. Please check for accuracy.

AQ5: Please check quality of figure reproduction. Your submitted pdf files were used, and they may be adequate. If any need replacement, you can attach original files to the editor by email, when you return materials.

AQ6: Please check whether corresponding author information is OK as typeset. 